\title{
Development of Single-Transistor-Control LDO Based on Flipped Voltage Follower for SoC
}

\author{
Tsz Yin Man, Student Member, IEEE, Ka Nang Leung, Senior Member, IEEE, Chi Yat Leung, \\ Philip K. T. Mok, Senior Member, IEEE, and Mansun Chan, Senior Member, IEEE
}

\begin{abstract}
The design issues of a single-transistor-control (STC) low-drop-out (LDO) based on flipped voltage follower is discussed in this paper, in particular the feedback stability at different conditions of output capacitors, equivalent series resistances (ESRs) and load current. Based on the analysis, an STC LDO was implemented in a standard 0.35- $\mu \mathrm{m}$ CMOS technology. It is proven experimentally that the LDO provides stable voltage regulation at a variety of output-capacitor/ESR conditions and is also stable in no output capacitor condition. The preset output voltage, minimum unregulated input voltage, maximum output current at a dropout voltage of $200 \mathrm{mV}$, ground current and active chip area are $1 \mathrm{~V}, 1.2 \mathrm{~V}, 50$ $\mathrm{mA}, 95 \mu \mathrm{A}$, and $140 \mu \mathrm{m} \times 320 \mu \mathrm{m}$, respectively. The full-load transient response in the no output capacitor case is faster than a micro second and is about $300 \mathrm{~ns}$.
\end{abstract}

Index Terms-Flipped voltage follower (FVF), low drop out (LDO), loop gain and power management.

\section{INTRODUCTION}

$\mathbf{P}$ OWER management is a timely and essential research area, enabling advancement of system-on-chip (SoC) technology. The development of high-performance integrated voltage regulators, in terms of accuracy, power efficiency, response time, silicon area, and off-chip component free feature, is undoubtedly vital to the success of SoC. To enable these requirements, a low drop out (LDO) with single-transistor control (STC) based on the flipped voltage follower (FVF) [1]-[3], with emphasis on the circuit theory for successful implementation, is presented in this paper [4].

It is well known that generic LDO structure suffers from unavoidable tradeoffs between the accuracy and feedback stability [5], [6]. A high loop gain, which results in improved steady-state regulation, degrades close-loop stability, so that different methodologies such as an advanced pole-zero can-

Manuscript received April 2, 2007; revised July 26, 2007. This work was supported by the Research Grant Council of Hong Kong SAR Government under project number HKUST 617705. This paper was recommended by Associate Editor T. B. Tarim.

T. Y. Man, was with the Department of Electronic and Computer Engineering, The Hong Kong University of Science and Technology, Clear Water Bay, Hong Kong. He is now with Marvell Hong Kong Ltd., Hong Kong (e-mail: scottie@marvell.com).

P. K. T. Mok and M. Chan are with the Department of Electronic and Computer Engineering, The Hong Kong University of Science and Technology, Clear Water Bay, Hong Kong (e-mail: eemok@ece.ust.hk; mchan@ece.ust.hk).

K. N. Leung and C. Y. Leung were with the Department of Electronic and Computer Engineering, The Hong Kong University of Science and Technology, Clear Water Bay, Hong Kong. They are now with the Department of Electronic Engineering, The Chinese University of Hong Kong, Shatin, Hong Kong (e-mail: knleung@ee.cuhk.edu.hk; cyleung@ee.cuhk.edu.hk).

Digital Object Identifier 10.1109/TCSI.2008.916568 cellation scheme in [5], a load-dependent reference voltage concept in [6], pole-splitting schemes in [7]-[10], were proposed. Recently, a super source follower [11], in form of FVF [1]-[3], has been applied to the designs of a buffer [12] and a power stage [13] in LDO. The main advantage of the FVF is the reduced output impedance due to shunt feedback connection [11], which is the key for obtaining good regulation and achieving frequency compensation. However, there are, in fact, many design issues have to be studied when using the FVF as a power stage. The studies in [1]-[3] do not focus on LDO design, and the application of the FVF in [12] is not for the power stage. In addition to the impedance control in [13], loop stability is undoubtedly a key issue needed to be analyzed in detail, especially when using different combinations of output capacitor and ESR values [14], [15] or when operating in the no output-capacitor (no-capacitor) condition [7], [8].

With regard to the above considerations, this paper intends to provide a detailed study on the stability of an LDO based on the FVF for different capacitors, equivalent series resistances (ESRs) and load conditions. No-capacitor condition for SoC applications is one of the topics that will be discussed in this paper.

The organization of this paper is as follows: Section II will introduce the STC-LDO structure and discuss the voltage regulation based on its local series-shunt feedback [11]. Section III will study the stability of the STC-LDO. The stability of the cases with the presence of an off-chip capacitor and no capacitor will be analyzed. Section IV will cover the load transient response and design requirements for fast response. Experimental results will be included in Section V.

\section{StRUCTURE AND WORKING PRINCIPLE OF STC-LDO}

The complete structure of a STC-LDO, including the required control-voltage generator, is shown in Fig. 1. In particular, the STC-LDO is mainly composed of $M_{P}$ (the power pMOSFET to deliver load current from the supply to the output), $M_{C}$ (the control transistor) and a current source $I_{\text {BIAS }}$. The parasitic resistances and capacitances are included in the analysis in Section III. There is an off-chip capacitor $C_{\text {OUT }}$ with ESR of $R_{E}$. $I_{\text {OUT }}$ models the loading circuit.

The source terminal of $M_{C}$ is the sensing terminal of the common-gate amplifier $M_{C}$. When $V_{\text {OUT }}$ varies, $M_{C}$ provides an error voltage at its drain to control the gate voltage of $M_{P}$. This mechanism controls the amount of drain current delivered by $M_{P}$ to regulate $V_{\mathrm{OUT}}$. The control voltage $V_{\mathrm{CTRL}}$ is to provide the preset $V_{\text {OUT }}$, according to the relationship

$$
V_{\mathrm{OUT}}=V_{\mathrm{CTRL}}+V_{\mathrm{SGC}}
$$




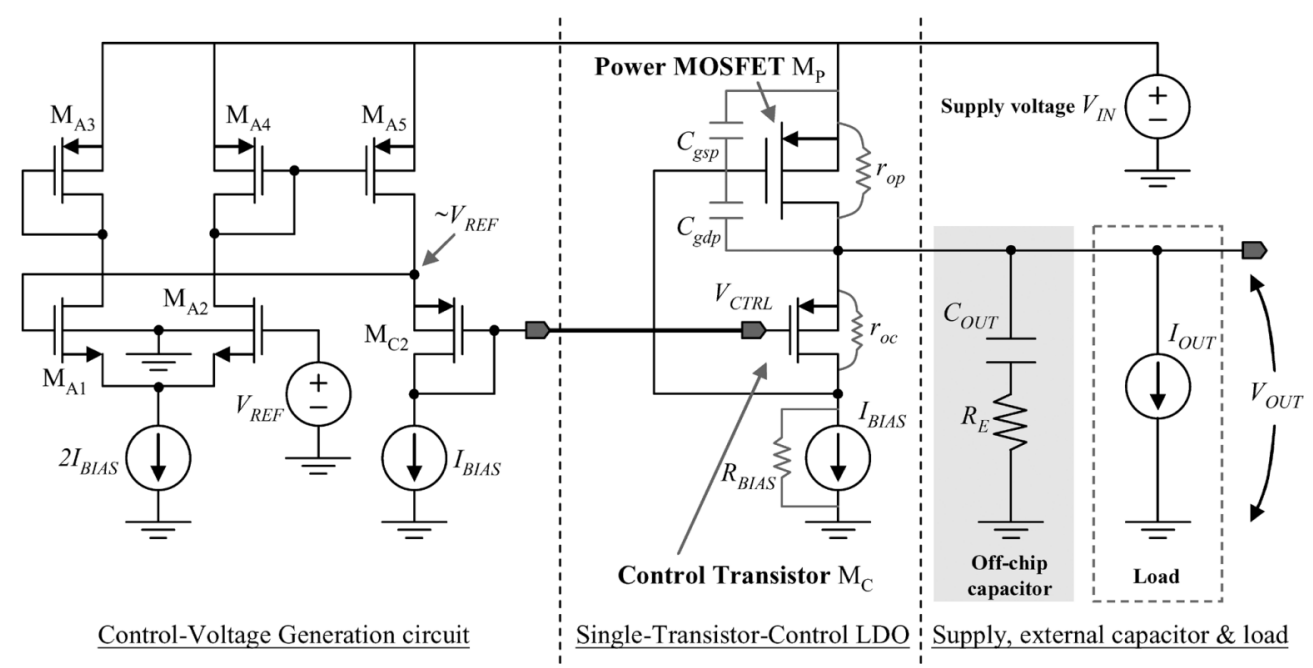

Fig. 1. Proposed STC LDO with the control-voltage generation circuit.

where $V_{\mathrm{SGC}}$ is the source-gate voltage of $M_{C} . V_{\mathrm{SGC}}$ is a constant and is independent of $I_{\text {OUT }}$ since it is biased constantly by $I_{\text {BIAS }}$

It is obvious from (1) that $V_{\text {OUT }}$ cannot be controlled independently of temperature and process variations due to $V_{\mathrm{SGC}}$. Therefore, a control-voltage generator is designed in Fig. 1 to overcome this problem.

The control-voltage generator is basically a simple amplifier in unity-gain configuration, except an additional transistor $M_{C 2}$ in diode connection biased by $I_{\mathrm{BIAS}}$ (same bias level of $M_{C}$ ) is inserted at the output stage. By providing a low tempco reference voltage $V_{\mathrm{REF}}$ (e.g., bandgap voltage reference) at the input of the unity-gain buffer, this $V_{\mathrm{REF}}$ will be re-generated at the output of the buffer. Thus, $V_{\mathrm{CTRL}}$ is given by

$$
V_{\mathrm{CTRL}}=V_{\mathrm{REF}}-V_{\mathrm{SGC} 2}
$$

where $V_{\mathrm{SGC} 2}$ is the source-gate voltage of $M_{C 2}$. Since $V_{\mathrm{SGC} 2}=V_{\mathrm{SGC}}\left(M_{C}\right.$ and $M_{C 2}$ are of the same size and of the same bias condition), the following relationship is achieved:

$$
V_{\mathrm{OUT}}=V_{\mathrm{REF}} .
$$

Scalable $V_{\mathrm{REF}}$ could provide an adjustable $V_{\text {OUT }}$ accordingly [11]. The channel length of $M_{C}$ and $M_{C 2}$ are suggested to be long such that channel modulation effect due to different $V_{S D}$ can be minimized. A decoupling capacitor is suggested to be connected between the voltage node $V_{\mathrm{CTRL}}$ and the ground for eliminating voltage spikes at $V_{\text {OUT }}$ through charge re-distribution of $C_{g d}$ of $M_{C}$ during the fast transient response for achieving better dynamics accuracy. In addition, the suggested decoupling capacitor helps to lessen noise injection to the STCLDO.

This STC-LDO is simpler than the one reported in [13]. It can be easily observed that the structure in [13] is suitable for wide supply-voltage range. However, LDO is designed for voltage regulation at a small dropout voltage (even at the maximum $I_{\text {OUT }}$ ) for maximizing the power-conversion efficiency. Therefore, the typical application of the LDO is to provide a regulated

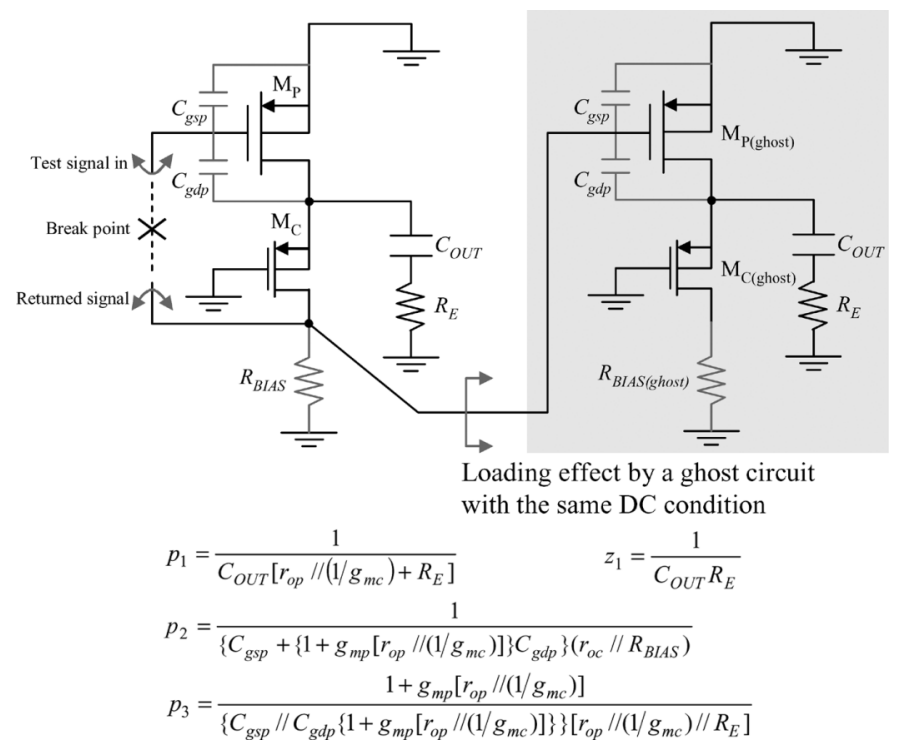

Fig. 2. Loop gain analysis by including loading effect.

voltage from a close-to-output supply voltage or as a post-regulator. The STC-LDO is well-suited for these applications, due to its extremely simple structure. The regulation range of the STC-LDO is given by

$$
V_{\mathrm{IN}}<V_{\mathrm{OUT}}+V_{\mathrm{SGP}}-V_{\mathrm{SDC}(\mathrm{sat})}
$$

where $V_{\mathrm{SDC}(\mathrm{sat})}$ is the saturation voltage of $M_{C}$. From (4), the regulation range counting from $V_{\mathrm{OUT}}$ is about one $V_{\mathrm{SGP}}$, which is generally sufficient for the aforementioned applications.

The mechanism of voltage regulation is explained here. Supposing $V_{\text {OUT }}$ is lower than the preset value, $V_{\mathrm{SGC}}$ will be enforced to be reduced such that the gate voltage of $M_{P}$ decreases due to the non-inverting voltage gain of a common-gate amplifier. The increase of $V_{\mathrm{SGP}}$ causes more drain current to be sourced to the load, as well as to the output capacitor. $V_{\text {OUT }}$ therefore increases. Similarly, when $V_{\text {OUT }}$ is higher than the preset value, $V_{\mathrm{SGP}}$ is reduced and $M_{P}$ delivers less drain current 


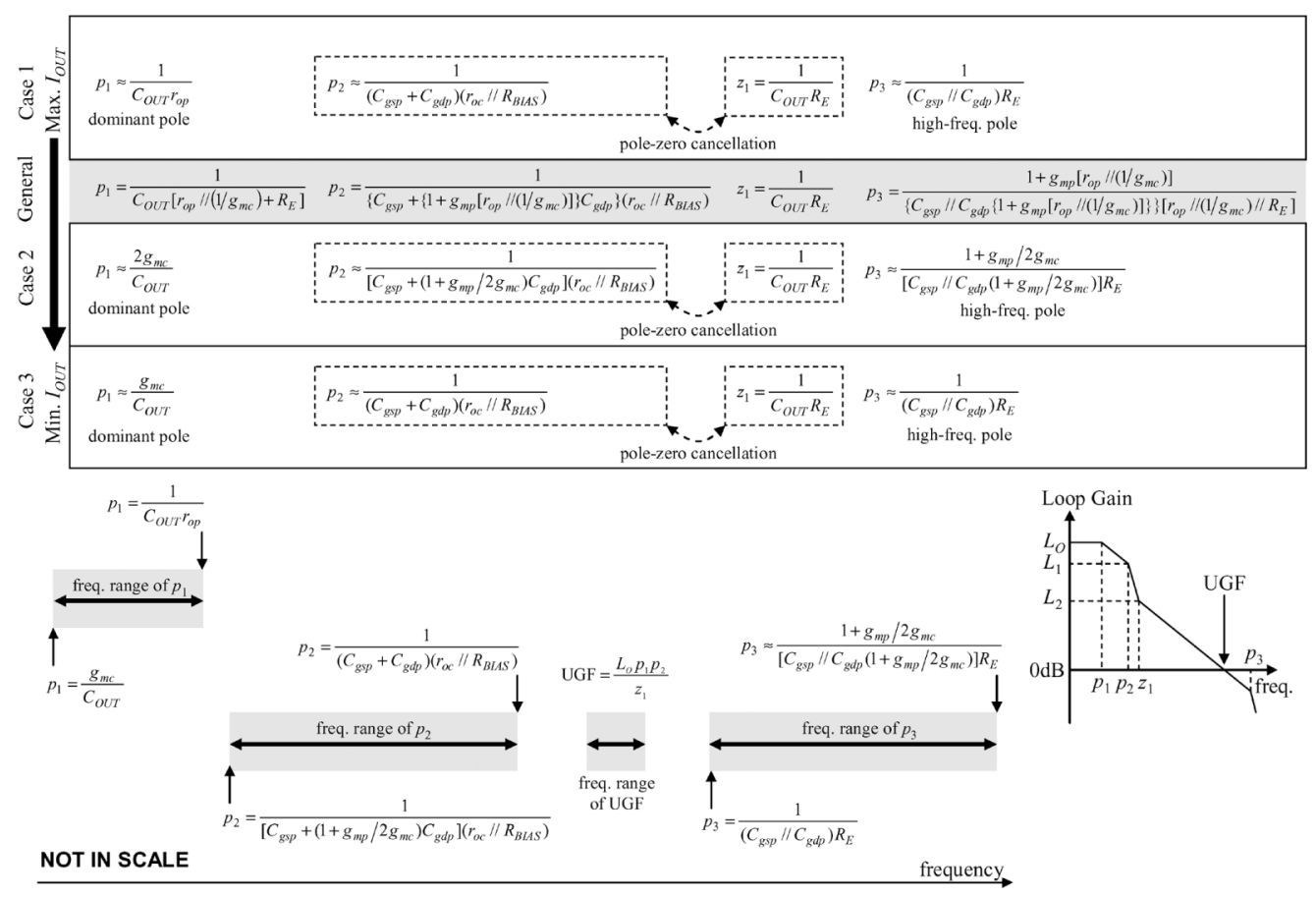

Fig. 3. Pole-zero analysis of the loop-gain transfer function of the STC-LDO with an off-chip capacitor with $R_{E}$ not large.

to cause a decrease of $V_{\text {OUT }}$. This continuous feedback results in the voltage regulation of STC-LDO at the preset voltage defined by $V_{\mathrm{REF}}$.

The line and load regulations of a LDO are given by [5], [7]

$$
\begin{aligned}
\text { Load regulation } & =\frac{\Delta V_{\mathrm{OUT}}}{\Delta I_{\mathrm{OUT}}}=\frac{R_{O}}{1+A_{\mathrm{EA}} G_{m} R_{O}} \\
& \approx \frac{1}{A_{\mathrm{EA}} G_{m}} \\
\text { Line regulation } & =\frac{\Delta V_{\mathrm{OUT}}}{\Delta V_{\mathrm{IN}}}=\frac{G_{m} R_{O}}{1+A_{\mathrm{EA}} G_{m} R_{O}} \\
& \approx \frac{1}{A_{\mathrm{EA}}}
\end{aligned}
$$

where $A_{\mathrm{EA}}$ is the voltage gain of the error amplifier in LDO; $G_{m}$ and $R_{O}$ are the transconductance and open-loop output resistance of the power pMOSFET. $R_{O}$ in STC-LDO is reduced by $1 / g_{m c}$. In general, $G_{m} R_{O}>1$ for low-to-moderate $I_{\text {OUT }}$. The approximations in both (5) and (6) are valid. This shows that the STC-LDO behaves similar to the conventional LDO. When $I_{\mathrm{OUT}}$ is high, $R_{O}$ will be dominated by $r_{\mathrm{op}}$ but not $1 / g_{m c}$. However, although $G_{m} R_{O}$ may not be greater one and the approximations in (5) and (6) are no longer valid, both load and line regulations of the STC-LDO are just same as those of the conventional LDO. Therefore, the key to improve both load and line regulations is to develop a high-gain error amplifier, while unaffecting the closed-loop stability.

\section{STABILITY OF STC-LDO}

The previously-stated voltage-regulation mechanism relies much the stability of the built-in local negative feedback of the STC-LDO. In this section, two cases will be studied. The first case is the STC-LDO with an off-chip capacitor. Different combinations of the loading condition and capacitor/ESR will be studied. The second case is the no-capacitor condition.

\section{A. Presence of Off-Chip Capacitor}

Fig. 2 shows the equivalent small-signal circuit of the STCLDO. Loop-gain analysis is done by breaking the feedback loop at the gate of $M_{P}$. The loading effect due by $M_{P}$ is included by a "ghost" circuit.

The transistor sizes of $M_{P \text { (ghost) }}$ and $M_{C \text { (ghost) }}$ are exactly equal to those of $M_{P}$ and $M_{C}$, respectively. Moreover, the dc bias of $M_{P \text { (ghost) }}$ and $M_{C \text { (ghost) }}$ are same as that of $M_{P}$ and $M_{C}$ as well. From the analysis, it is found that there are three left-half-plane (LHP) poles [shown in (7a)-(7c), at the bottom of the next page], and one LHP zero as follows.

$$
z_{1}=\frac{1}{C_{\mathrm{OUT}} R_{E}}
$$

The poles are $I_{\mathrm{OUT}}$-dependent, since different $I_{\mathrm{OUT}}$ vary $g_{m p}\left(\propto \sqrt{I_{\text {OUT }}}\right)$ and $r_{\mathrm{op}}\left(\propto 1 / I_{\text {OUT }}\right)$. In particular, $r_{\mathrm{op}}$ may be smaller or larger than $1 / g_{m c}$ and $R_{E}$ in different $I_{\text {OUT }}$. Moreover, from (7b), Miller effect happens to $C_{g d p}$ such that $p_{2}$ is pushed to a lower frequency. Fig. 3 summaries the relationship of the poles and zero in different $I_{\mathrm{OUT}}$. It is highlighted that this analysis is for $R_{E}$ not large. The boundary of this definition relates to $r_{\mathrm{op}}$. For example, when $M_{P}$ operates in the saturation region in low $I_{\text {OUT }}$ condition, $r_{\mathrm{op}}$ is definitely larger than $R_{E}$. When $M_{P}$ is in the dropout region, $r_{\mathrm{op}}$ is reduced. The worst case is when the $M_{P}$ operates in the linear region, and $r_{\mathrm{op}}$ can be simply found by $V_{\mathrm{SDP}} / I_{\mathrm{SDP}}$. Therefore, when the dropout voltage is $200 \mathrm{mV}$ and $I_{\mathrm{OUT}}=50 \mathrm{~mA}, r_{\mathrm{op}}$ is $4 \Omega$. Based on this study, $r_{\mathrm{op}}$ is generally larger than $R_{E}$, which is about $100 \mathrm{~m} \Omega$ for capacitance in micro-Farad up to a few ohms for capacitance in nanofarad. In general, a large $R_{E}$ is not preferred since this introduces large voltage spikes in the transient response [16]. 
The study is considered when the $I_{\mathrm{OUT}}$ decreases from the maximum to zero (i.e., $I_{\text {BIAS }}$ ). The equations in (7a) to (7c) are modified at different $I_{\mathrm{OUT}}$. There are three possible cases:

1) Case 1: $I_{\mathrm{OUT}}=$ maximum or high: When $I_{\mathrm{OUT}}$ is very large, $r_{\mathrm{op}}$ is much smaller than $1 / g_{m c}$ but is larger than $R_{E}$. Therefore, $p_{1}$ is dominated by $r_{\mathrm{op}}$, and the Miller effect at $p_{2}$ is negligible. From Fig. 3, $p_{2}$ is cancelled by $z_{1}$ within one decade of frequency. $p_{1}$ becomes the dominant pole. Since $p_{3}$ is a function of $C_{g s p}, C_{g d p}$ and $R_{E}, p_{3}$ is located at a frequency higher than the unity-gain frequency (UGF) of the loop gain. Thus, the loop gain basically has a single pole, and it is absolutely stable [11].

2) Case 2: $I_{\mathrm{OUT}}=$ moderate: When $I_{\mathrm{OUT}}$ is lower and is at a level such that $r_{\mathrm{op}}=1 / g_{m c},(7 \mathrm{a})-(7 \mathrm{c})$ are reduced to the forms in Fig. 3. $p_{1}$ becomes a constant. The change of $I_{\mathrm{OUT}}$, in principle, does not affect $p_{1}$. The Miller effect to $p_{2}$ is also not significant due to the small $g_{m p}$, but $p_{2}$ is located at a relatively lower frequency than that in Case 1 . Similar to Case $1, p_{3}$ is at a high frequency. Thus, $z_{1}$ is designed to cancel $p_{2}$ within one decade of frequency. $p_{1}$ is the dominant pole, and $p_{3}$ is higher than UGF. The feedback loop is stable.

3) Case 3: $I_{\mathrm{OUT}}=0$ : When $I_{\mathrm{OUT}}=0$, the drain current of $M_{P}$ is equal to $I_{\mathrm{BIAS}}$ (see Fig. 1). $r_{\mathrm{op}}$ is much larger than $1 / g_{m c}$, and $g_{m p}$ is close to $g_{m c}$ (as $M_{P}$ is in deep subthreshold region) such that Miller effect is not significant. Similar to Case 2 , the change of $I_{\mathrm{OUT}}$, in principle, does not affect $p_{1}$. However, $p_{2}$ moves to a relatively higher frequency than that in Case 2. This illustrates $p_{2}$ is bounded due to the STC-LDO structure. As a result, the design of $z_{1}$ by $R_{E}$ is much easier than typical LDO, since the range of $p_{2}$ is bounded. The only requirement of $R_{E}$ is that $z_{1}$ should be designed within the range of $p_{2}$ (shown in Fig. 3) to achieve the best cancellation of $p_{2}$ for the stability of the STC-LDO.

From the discussion, it is found that $p_{1}$ is load-independent in principle when $I_{\mathrm{OUT}}$ is reduced. The loop bandwidth at the moderate-to-zero load conditions is much better than the conventional LDO [17]. In addition, $p_{2}$ is bounded so that the requirement of $R_{E}$ is not as harsh as in the conventional LDO design.

In case of a large ESR such that $R_{E} \ll r_{\mathrm{op}}$ and $1 / g_{m c}$ in any $I_{\text {OUT }}$ condition, it is trivial from (7a) and (7d) that $p_{1}$ is exactly equal $z_{1}$ for perfectly pole-zero cancellation, i.e.,

$$
p_{1}=z_{1}=\frac{1}{C_{\mathrm{OUT}} R_{E}} .
$$

Both $p_{2}$ and $p_{3}$ are independent of $C_{\text {OUT }} \cdot p_{2}$ will be the dominant pole, and $p_{3}$ is always at a frequency higher than UGF. It
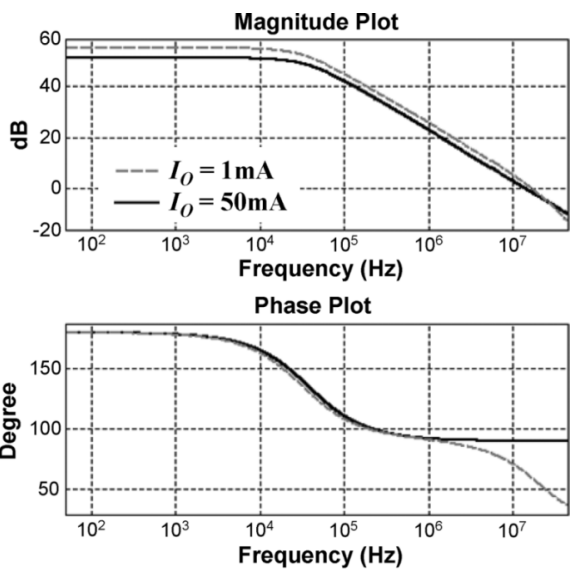

(a)

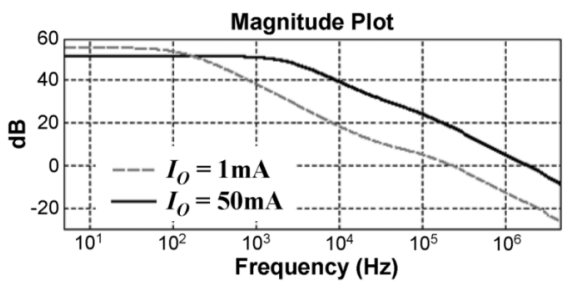

Phase Plot

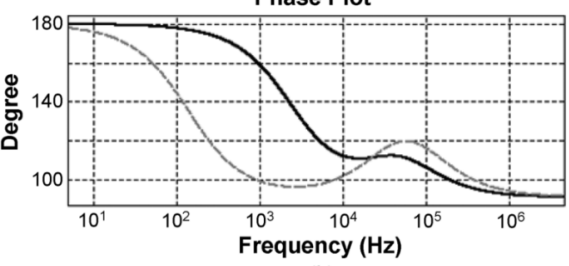

(b)

Fig. 4. Simulated loop gain (a) no output capacitor (b) $C_{\mathrm{OUT}}=4.7 \mu \mathrm{F}$ and $R_{E}=1 \Omega$

can be proven by comparing the effective capacitance and the effective resistance of both $p_{2}$ and $p_{3}$. As a result, the stability of STC-LDO is achieved when $R_{E}$ is very large.

It is noted that the UGF of Case 1, 2, and 3 can be found by the magnitude plot in Fig. 3 using gain-bandwidth product. Therefore, $L_{O} p_{1}=L_{1} p_{2}, L_{1} p_{2}^{2}=L_{2} z_{1}^{2}$ and $L_{2} z_{1}=\mathrm{UGF}$, the expression of UGF $=L_{O} p_{1} p_{2} / z_{1}$ can be obtained. The UGF is obviously not a constant since $L_{O}, p_{1}$ and $p_{2}$ are loaddependent.

\section{B. No-Output Capacitor}

When there is no off-chip capacitor, there is just a parasitic capacitance due by the routing to the load circuit. As a result, it is reasonable to claim $C_{\mathrm{OUT}} \rightarrow 0$ and $R_{E}=0 . p_{1}$ in (7a) locates to a very high frequency when $C_{\text {OUT }} \rightarrow 0$

$$
\begin{aligned}
p_{1} & =\frac{1}{C_{\mathrm{OUT}}\left[r_{\mathrm{op}} / /\left(1 / g_{m c}\right)+R_{E}\right]} \\
p_{2} & =\frac{1}{\left\{C_{g s p}+\left\{1+g_{m p}\left[r_{\mathrm{op}} / /\left(1 / g_{m c}\right)\right]\right\} C_{g d p}\right\}\left(r_{o c} / / R_{\mathrm{BIAS}}\right)} \\
p_{3} & =\frac{1+g_{m p}\left[r_{\mathrm{op}} / /\left(1 / g_{m c}\right)\right]}{\left\{C_{g s p} / / C_{g d p}\left\{1+g_{m p}\left[r_{\mathrm{op}} / /\left(1 / g_{m c}\right)\right]\right\}\right\}\left[r_{\mathrm{op}} / /\left(1 / g_{m c}\right) / / R_{E}\right]}
\end{aligned}
$$




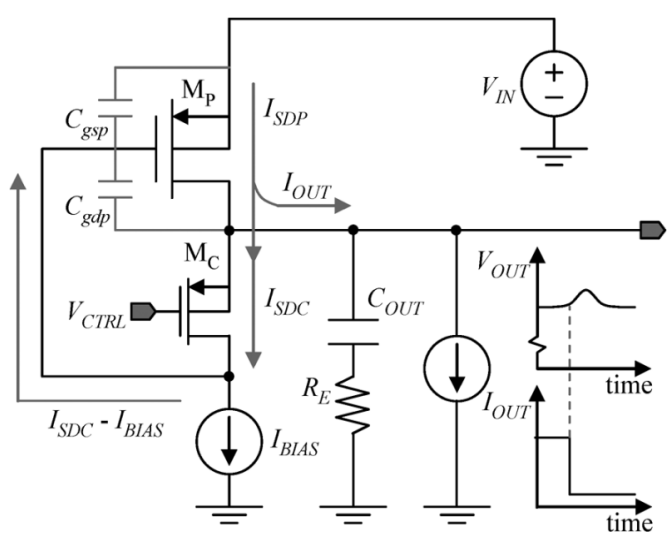

(a)

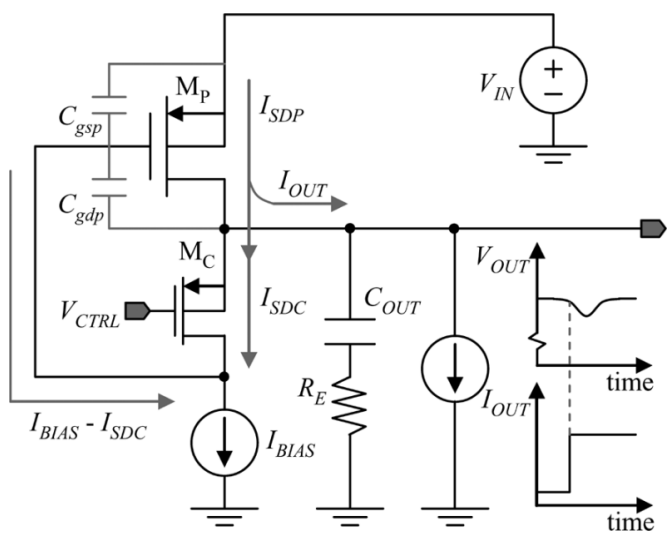

(b)

Fig. 5. Analysis of the load transient response. (a) Step-down load. (b) Step-up load.

and $z_{1}$ in $(7 \mathrm{~d})$ vanishes. By re-written $p_{2}$ into $p_{1(n c)}, p_{3}$ into $p_{2(n c)}$ and $p_{1}$ into $p_{3(n c)}$, the remaining two poles are given by (9a)-(9c), at the bottom of the page. From (9a) and $(9 \mathrm{~b})$, it is obviously that $p_{2(n c)}$ locates at a much higher frequency than $p_{1(n c)}$ at different $I_{\mathrm{OUT}}$. Moreover, when the STC-LDO is used to power-up a local, on-chip circuit block individually, the equivalent load capacitance from the small circuit block is not much. It is just about $\sim 20 \mathrm{pF}$ at most. Assuming $g_{m c}=400 \mu \mathrm{A} / \mathrm{V}$ and $r_{\mathrm{op}}$ is larger than $1 / g_{m c}$ (the worst case), the corresponding position of $p_{3(n c)}$ is about $3 \mathrm{MHz}$ and is typically higher than the UGF of the loop bandwidth of the STC-LDO when $C_{\text {OUT }}=20 \mathrm{pF}$. Therefore, STC-LDO is absolutely stable in no-capacitor condition. Moreover, $p_{1}$ is a function of the small parasitic capacitances. The loop response in no-capacitor condition is fast.

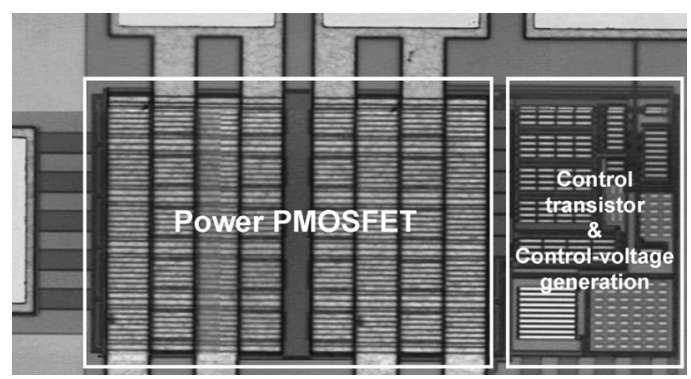

Fig. 6. Micrograph of the STC-LDO.

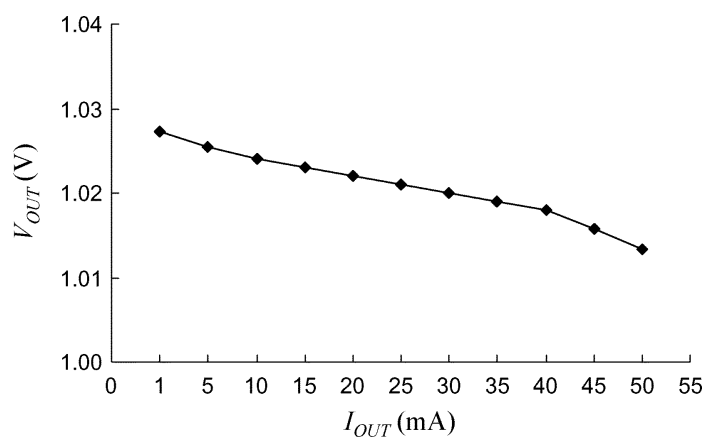

Fig. 7. Measured load regulation of STC-LDO at $V_{\mathrm{IN}}=1.2 \mathrm{~V}$.

The proposed LDO in Fig. 1 have been simulated using the BSim3v3 models of a $0.35-\mu \mathrm{m}$ CMOS process, provided by Austria Mikro System (AMS) Group, Austria. Fig. 4 shows the loop-gain simulations with and without the output capacitor. From the simulation, the proposed LDO has phase margin of more than $60^{\circ}$ when $I_{\mathrm{OUT}}=1 \mathrm{~mA}$ and $I_{\mathrm{OUT}}=50 \mathrm{~mA}$.

\section{TRANSIENT RESPONSE OF STC-LDO}

The load transient response of STC-LDO can be studied by Fig. 5. The cases of step-down load and step-up load are analyzed in this section. A design condition of the control transistor will be derived after the analysis.

1) Case 1: Step-Down Load [Refer to Fig. 5(a)]: When $I_{\mathrm{OUT}}$ decreases rapidly, the drain current of $M_{P}, I_{\mathrm{SDP}}$, cannot stop instantaneously. The output capacitor is over-charged and the capacitor voltage exceeds the preset $V_{\mathrm{OUT}}$. The $V_{\mathrm{SGC}}$ is thus increased, and this causes a larger $I_{\mathrm{SDC}}$ happened. There is an excess current of amount of $I_{\mathrm{SDC}}-I_{\mathrm{BIAS}}$ to charge the gate capacitance of $M_{P}$ for increasing the gate voltage such that $M_{P}$ will deliver less current to the load. The overshoot is then settled.

$$
\begin{aligned}
p_{1(n c)} & =\frac{1}{\left\{C_{g s p}+\left\{1+g_{m p}\left[r_{\mathrm{op}} / /\left(1 / g_{m c}\right)\right]\right\} C_{g d p}\right\}\left(r_{o c} / / R_{\mathrm{BIAS}}\right)} \\
p_{2(n c)} & =\frac{1+g_{m p}\left[r_{\mathrm{op}} / /\left(1 / g_{m c}\right)\right]}{\left\{C_{g s p} / / C_{g d p}\left\{1+g_{m p}\left[r_{\mathrm{op}} / /\left(1 / g_{m c}\right)\right]\right\}\right\}\left[r_{\mathrm{op}} / /\left(1 / g_{m c}\right) / / R_{E}\right]} \\
p_{3(n c)} & =\frac{1}{C_{\mathrm{OUT}}\left[r_{\mathrm{op}} / /\left(1 / g_{m c}\right)\right]}
\end{aligned}
$$




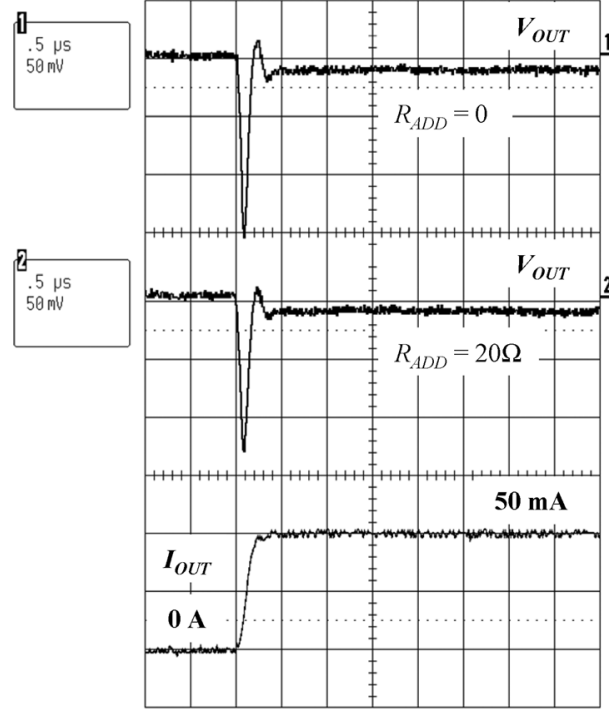

(a)
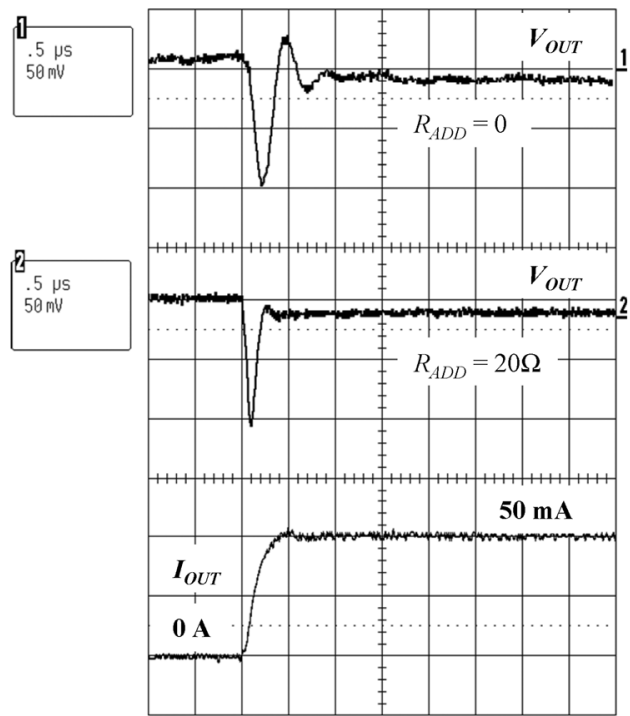

(b)

Fig. 8. Measured load transient responses an off-chip capacitor of 1 and $47 \mathrm{nF}$, where $R_{\mathrm{ADD}}$ is the added series resistance. (a) $C_{\mathrm{OUT}}=1 \mathrm{nF}$ and $R_{E} \approx 4.4 \Omega$. (b) $C_{\text {OUT }}=47 \mathrm{nF}$ and $R_{E} \approx 2.2 \Omega$.
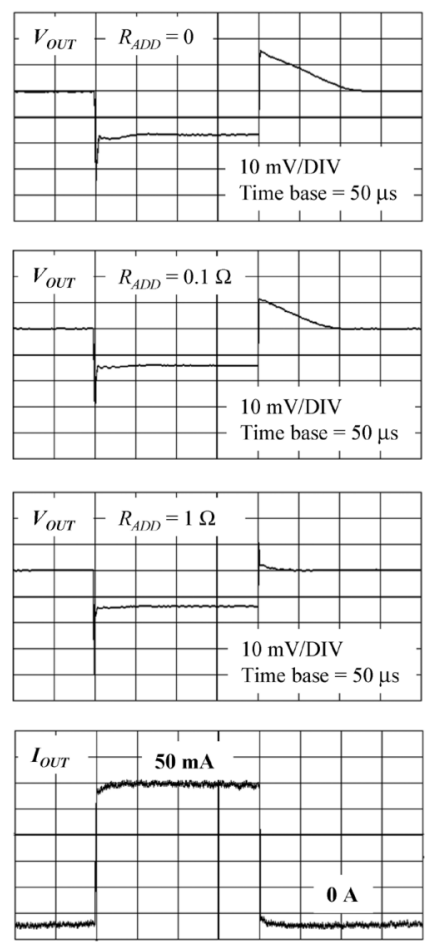

(a)
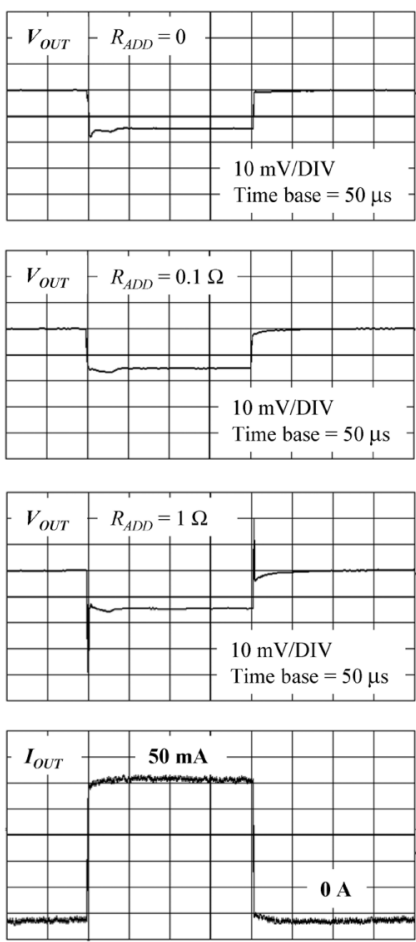

(b)
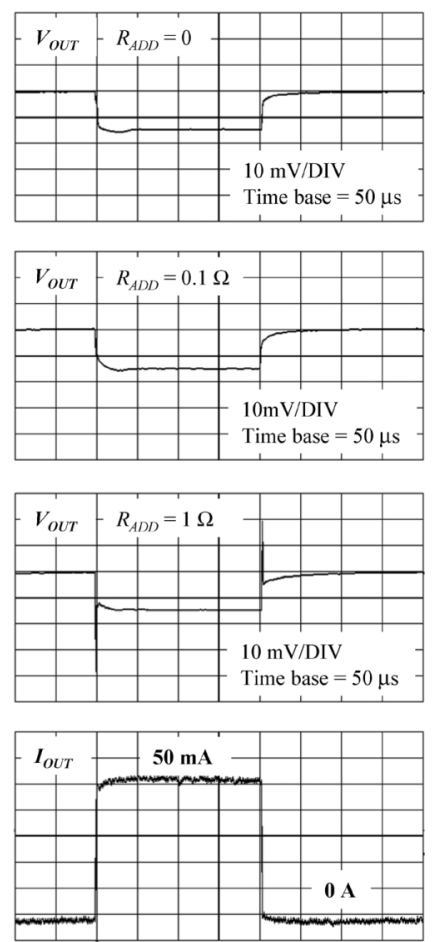

(c)

Fig. 9. Measured load transient responses with an off-chip capacitor of $1,4.7$, and $10 \mu \mathrm{F}$, where $R_{\mathrm{ADD}}$ is the added series resistance (full view). (a) $C_{\mathrm{OUT}}=1 \mu \mathrm{F}$ and $R_{E} \approx 16 \mathrm{~m} \Omega$. (b) $C_{\text {OUT }}=4.7 \mu \mathrm{F}$ and $R_{E} \approx 32 \mathrm{~m} \Omega$. (c) $C_{\text {OUT }}=10 \mu \mathrm{F}$ and $R_{E} \approx 32 \mathrm{~m} \Omega$.

2) Case 2: Step-Up Load [Refer to Fig. 5(b)]: Similarly, when $I_{\mathrm{OUT}}$ suddenly increases, $I_{\mathrm{SDP}}$ is not sufficient to supply the load. The output capacitor discharges and delivers current to the load. This causes drop of $V_{\text {OUT }}$. This drop causes the reduction of $V_{\mathrm{SGC}}$, and then $I_{\mathrm{SDC}}$ is reduced. The discharging current of the gate capacitance of $M_{P}$ is $I_{\mathrm{BIAS}}-I_{\mathrm{SDC}}$. Once the $V_{\mathrm{SGP}}$ increases, more drain current from $M_{P}$ will supply the load and charge $C_{\text {OUT }}$ back to the preset voltage.
From the case study, it reveals that $I_{\mathrm{SDC}}$ should be large for step-down load, while it should be small or even zero for step-up load. It is preferred that $I_{\mathrm{SDC}}$ is sensitive to $\Delta V_{\mathrm{SGC}}$. The design condition of $M_{C}$ for higher sensibility to $\Delta V_{\mathrm{SGC}}$ can be found by

$$
I_{\mathrm{SDC}}+\Delta I_{\mathrm{SDC}}=\frac{\mu_{p} C_{\mathrm{OX}}}{2}\left(\frac{W}{L}\right)\left(V_{\mathrm{SGC}}+\Delta V_{\mathrm{SGC}}-V_{\mathrm{TH}}\right)^{2}
$$



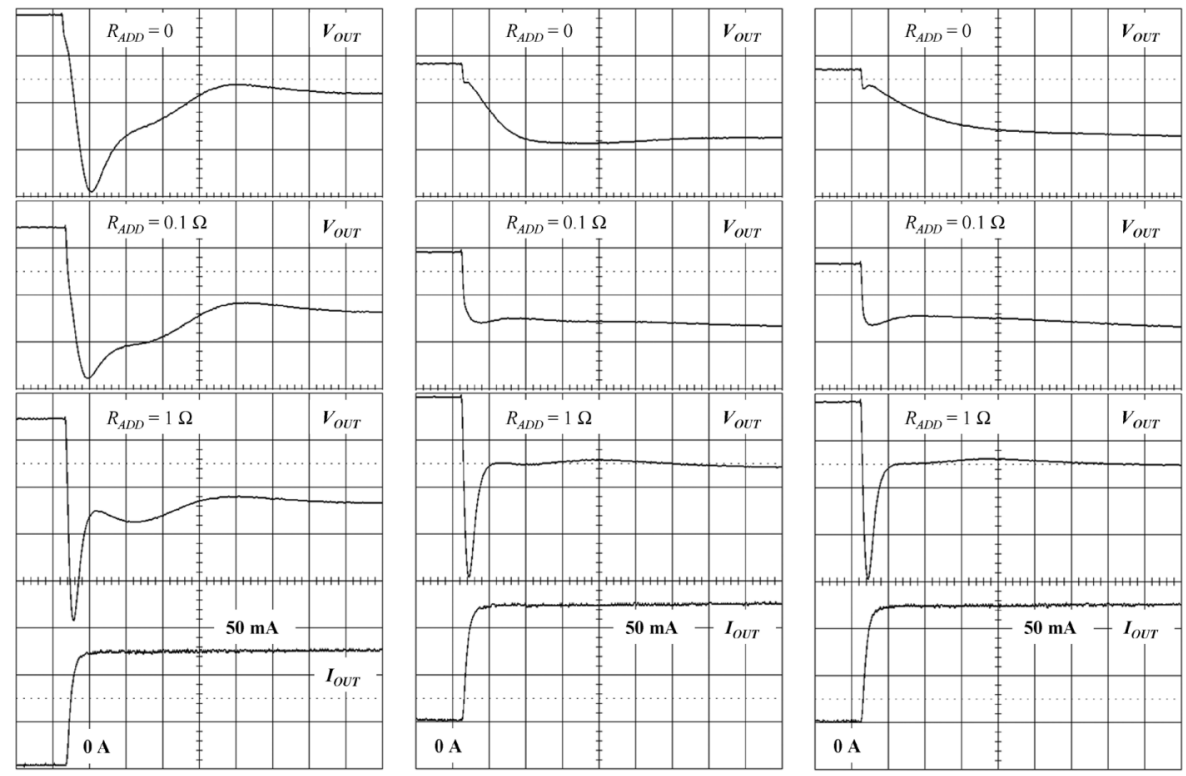

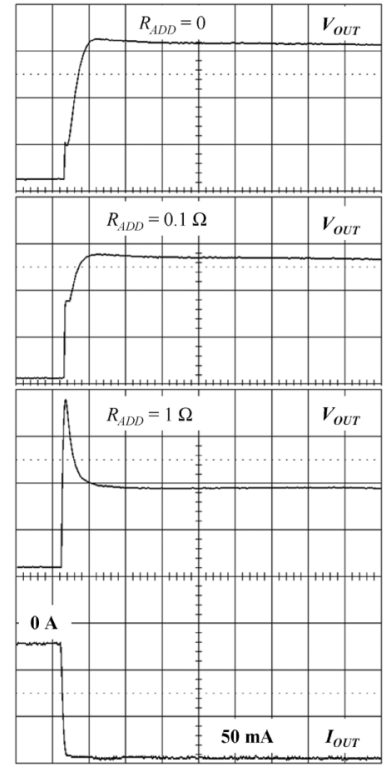

(a)

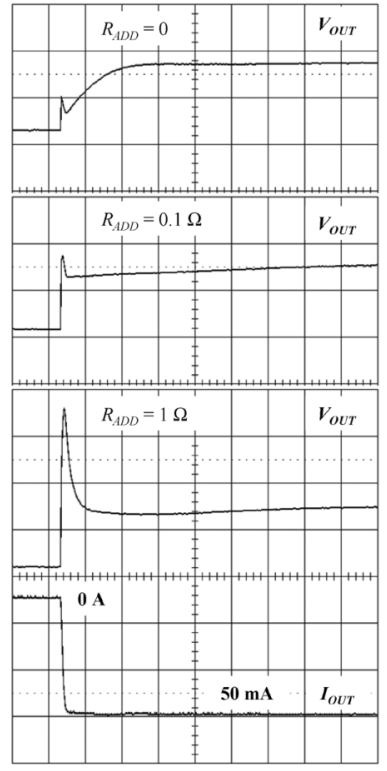

(b)

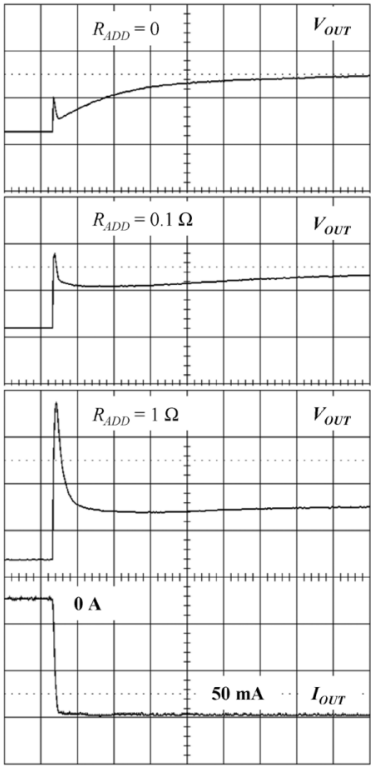

(c)

Fig. 10. Measured load transient responses with an off-chip capacitor of 1,4., and $10 \mu \mathrm{F}$, where $R_{\mathrm{ADD}}$ is the added series resistance (zoom-in view, time base $=$ $1 \mu \mathrm{s}$ and $V_{\mathrm{OUT}}$ displays at $10 \mathrm{mV} / \mathrm{DIV}$ ). (a) $C_{\mathrm{OUT}}=1 \mu \mathrm{F}$ and $R_{E} \approx 16 \mathrm{~m} \Omega$. (b) $C_{\mathrm{OUT}}=4.7 \mu \mathrm{F}$ and $R_{E} \approx 32 \mathrm{~m} \Omega$; (c) $C_{\mathrm{OUT}}=10 \mu \mathrm{F}$ and $R_{E} \approx 32 \mathrm{~m} \Omega$.

where $(W / L)$ is the aspect ratio of $M_{C}$. The variables in equation in (10) are extracted to

$$
\begin{aligned}
\Delta I_{\mathrm{SDC}}=\left[\mu_{p} C_{\mathrm{OX}}\left(\frac{W}{L}\right)(\right. & \left.\left.V_{\mathrm{SGC}}-V_{\mathrm{TH}}\right)\right] \Delta V_{\mathrm{SGC}} \\
& +\frac{\mu_{p} C_{\mathrm{OX}}}{2}\left(\frac{W}{L}\right) \Delta V_{\mathrm{SGC}}^{2} .
\end{aligned}
$$

From (11), it is found that the first term refers to the expected response such that a positive $\Delta V_{\mathrm{SGC}}$ results in a positive $\Delta I_{\mathrm{SDC}}$, and vice versa. However, the $\Delta V_{\mathrm{SGC}}^{2}$ term helps the positive $\Delta V_{\mathrm{SGC}}$ (step-down load case) but discourages the case with a negative $\Delta V_{\mathrm{SGC}}$ (step-up load case). Therefore, when both step-up and step-down cases are needed to improve, a larger overdrive voltage is preferred, while a small $W / L$ of $M_{C}$ is suggested.

\section{EXPERIMENTAL RESULTS}

The LDO regulator shown in Fig. 1 has been implemented in AMS 0.35- $\mu \mathrm{m}$ CMOS process. The chip micrograph is shown in Fig. 6. The chip area without testing pads is $140 \mu \mathrm{m} \times 320 \mu \mathrm{m}$. The regulator is designed to provide a load current of 0-50 mA with an output voltage of $1 \mathrm{~V}$ from a 1.2- to $1.5-\mathrm{V}$ supply. The dropout voltage is about $200 \mathrm{mV}$ at the maximum $I_{\text {OUT }}$ (the worst case). The ground current is $95 \mu \mathrm{A}$.

The measured line and load regulations at $V_{\mathrm{IN}}=1.2 \mathrm{~V}$ are $18 \mathrm{mV} / \mathrm{V}$ and $280 \mu \mathrm{V} / m A$, respectively. Fig. 7 shows the load regulation of the STC-LDO at $V_{\mathrm{IN}}=1.2 \mathrm{~V}$.

The measured load transient responses are shown in Figs. 8, 9 and 10. In Fig. 8, smaller-value capacitors ( $1 \mathrm{nF}$ and $47 \mathrm{nF})$ are used. Their measured ESRs are larger, as predicted and are about $4.4 \Omega$ (for $C_{\mathrm{OUT}}=1 \mathrm{nF}$ ) and $2.2 \Omega$ (for $C_{\mathrm{OUT}}=47 \mathrm{nF}$ ), 
TABLE I

SUMMARY OF EXISTING LDO STRUCTURES

\begin{tabular}{|c|c|c|c|c|c|c|c|c|}
\hline Ref. & $\begin{array}{c}\text { Power } \\
\text { Transistor }\end{array}$ & Features & Technology & $I_{Q}$ & $V_{I N}$ & IOUT(max) & Line Reg. & Load Reg. \\
\hline$[18]$ & \multirow[t]{2}{*}{ NMOS } & \multirow{2}{*}{$\begin{array}{l}\text { Charge-pumped gate } \\
\text { drive for low } \\
\text { dropout, cap-free. }\end{array}$} & $\begin{array}{c}0.8-\mu \mathrm{m} \\
\mathrm{BiCMOS}\end{array}$ & N/A & $4 \mathrm{~V}$ & $5 \mathrm{~mA}$ & $49 \mathrm{mV} / \mathrm{V}$ & $22 \mathrm{mV} / 7 \mathrm{~mA}$ \\
\hline$[21]$ & & & BiCMOS & $0.55 \mathrm{~mA}$ & $1.7-5.5 \mathrm{~V}$ & $150 \mathrm{~mA}$ & $0.01 \% / \mathrm{V}$ & $0.002 \% / \mathrm{mA}$ \\
\hline [19] & DMOS & $\begin{array}{l}\text { Zero } V_{T H} \text { device for } \\
\text { low dropout., } \\
\text { cap-free. }\end{array}$ & DMOS & $1 \mathrm{~mA}$ & N/A & $500 \mathrm{~mA}$ & N/A & N/A \\
\hline [20] & \multirow{8}{*}{ PMOS } & $\begin{array}{l}\text { Adaptively-biased } \\
\text { buffer for fast } \\
\text { transient response } \\
\text { and high efficiency. }\end{array}$ & $2-\mu \mathrm{m}$ CMOS & $23 \mu \mathrm{A}$ & $\geq 1 \mathrm{~V}$ & $50 \mathrm{~mA}$ & $4 \mathrm{mV} / 3.8 \mathrm{~V}$ & $19 \mathrm{mV} / 50 \mathrm{~mA}$ \\
\hline [5] & & \multirow{5}{*}{$\begin{array}{l}\text { Advanced frequency } \\
\text { compensation } \\
\text { scheme for loop } \\
\text { stability. Cap-free in } \\
\text { [7] and [8]. }\end{array}$} & $2-\mu \mathrm{m}$ CMOS & N/A & N/A & $50 \mathrm{~mA}$ & N/A & $12 \mathrm{mV} / 100 \mathrm{~mA}$ \\
\hline [7] & & & $0.6-\mu \mathrm{m}$ CMOS & $38 \mu \mathrm{A}$ & $1.5-4.5 \mathrm{~V}$ & $100 \mathrm{~mA}$ & \multicolumn{2}{|c|}{$0.25 \%$ in total } \\
\hline [8] & & & $0.35-\mu \mathrm{m}$ CMOS & N/A & $1.2-3.3 \mathrm{~V}$ & $100 \mathrm{~mA}$ & $344 \mu \mathrm{V} / \mathrm{V}$ & $388 \mu \mathrm{V} / \mathrm{mA}$ \\
\hline [9] & & & $0.5-\mu \mathrm{m}$ CMOS & $25 \mu \mathrm{A}$ & $3.3 \mathrm{~V}$ & $160 \mathrm{~mA}$ & N/A & $0.2 \mathrm{~V} / 160 \mathrm{~mA}$ \\
\hline [10] & & & $0.35-\mu \mathrm{m}$ CMOS & $53 \mu \mathrm{A}$ & $2-5 \mathrm{~V}$ & $150 \mathrm{~mA}$ & $0.143 \% / \mathrm{V}$ & $92.8 \mathrm{ppm} / \mathrm{mA}$ \\
\hline$[13]$ & & Simple LDO & 90-nm CMOS & $6 \mathrm{~mA}$ & $1.2 \mathrm{~V}$ & $100 \mathrm{~mA}$ & N/A & N/A \\
\hline $\begin{array}{l}\text { This } \\
\text { work }\end{array}$ & & $\begin{array}{l}\text { structure. Cap-free in } \\
\text { this paper. }\end{array}$ & $0.35-\mu \mathrm{m}$ CMOS & $95 \mu \mathrm{A}$ & $1.2-1.5 \mathrm{~V}$ & $50 \mathrm{~mA}$ & $18 \mathrm{mV} / \mathrm{V}$ & $280 \mu \mathrm{V} / \mathrm{mA}$ \\
\hline
\end{tabular}
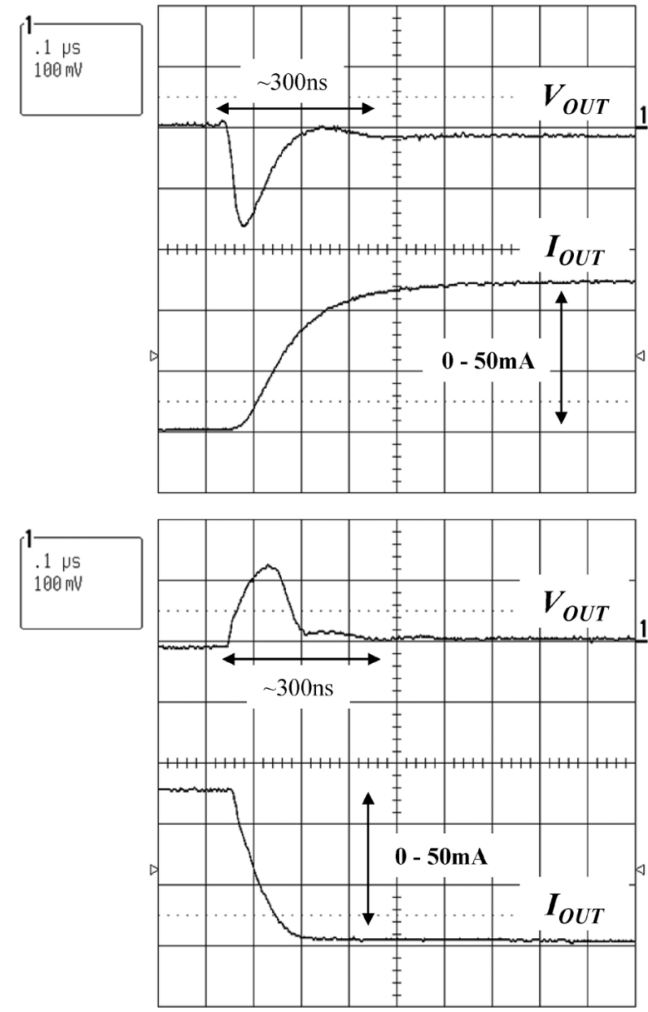

Fig. 11. Measured load transient response of the STC-LDO in no-capacitor condition.

respectively. It is obvious that the STC-LDO is stable in both cases. The settling time is as fast as $<1 \mu$ s. In Fig. 9 (Fig. 10 is the zoomed-in views), low-ESR capacitors are used for the measurement. $C_{\text {OUT }}=1 \mu \mathrm{F}, 4.7 \mu \mathrm{F}$ and $10 \mu \mathrm{F}$ with $R_{E}=16 \mathrm{~m} \Omega$ to $32 \mathrm{~m} \Omega$. In these measurement, $I_{\text {OUT }}$ changes between 0 and $50 \mathrm{~mA}$ in about $500 \mathrm{~ns}$. An added resistor $R_{\mathrm{ADD}}$ is connected in series with the off-chip capacitor to test the effects of the additional ESR with increment of decades. From Fig. 8, it is obvious that the STC-LDO is stable for those cases. The larger voltage spike when $I_{\mathrm{OUT}}=0 \rightarrow 50 \mathrm{~mA}$ and $50 \mathrm{~mA} \rightarrow 0$ is introduced
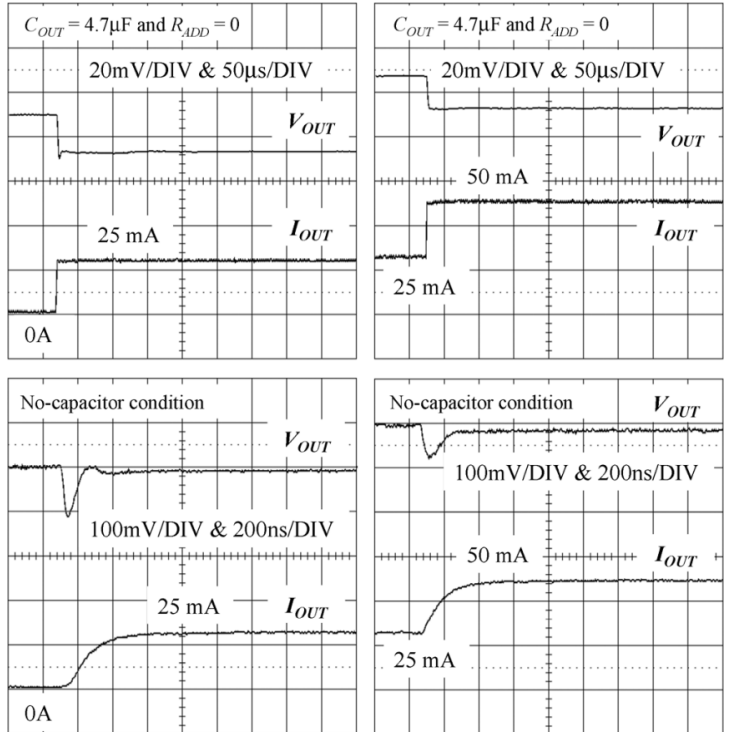

Fig. 12. More results of the load transient responses for the case with $C_{\mathrm{OUT}}=$ $4.7 \mu \mathrm{F}$ and the no-capacitor condition.

by the transient current flowing into the larger $R_{\mathrm{ADD}}$. Therefore, when the STC-LDO is stable, it is no reason to add $R_{\mathrm{ADD}}$.

For the no-capacitor case, a load transient response has been measured and is shown in Fig. 11. The measurement probe capacitance is about $20 \mathrm{pF}$, and $p_{3(n c)}$ is therefore higher than 1 $\mathrm{MHz}$, which is the estimated UGF of the loop bandwidth of the STC-LDO. From the result, the STC-LDO is again proven stable and responds fast to settle $V_{\text {OUT }}$ in about 300 ns. As a remark, there is no minimum load current requirement for the STC-LDO to be stable in the no-capacitor condition [7], [8].

More measurement results are included in Fig. 12 to prove the LDO is stable when the loading current changes from 0 to a moderate value and then to the maximum value. Two cases are specially tested: $C_{\mathrm{OUT}}=4.7 \mu \mathrm{F}$ and the no-capacitor condition. From the measurement waveforms, it is obviously that the LDO is stable. 


\section{CONCLUSION}

In this paper, the LDO regulator based on the FVF to reduce the output impedance has been described. The impacts of the structure have been examined. It has been discussed that the STC-LDO structure provides much better LDO stability than the conventional LDO. In particular,

1) The loop bandwidth at low load current is independent of the load current. It has wider bandwidth than the conventional LDO.

2) The requirement of the ESR of the output capacitor is relatively easier to achieve, comparing the conventional LDO. It is due to the fact that the load-dependent range of the second non-dominant pole is bounded.

3) The STC-LDO is absolutely stable for an output capacitor with a small or large ESR.

4) In addition, it is also absolutely stable in the no-capacitor condition. The loop bandwidth is wide as well for faster transient response.

Moreover, the load transient response has been investigated. A design condition to improve the sensitivity of the control transistor has been derived. Experimental results have proven the analysis and the stated arguments.

Finally, Table I summarizes the existing LDO structures using MOS technology, their features and the specifications. From this table, it reveals the recently developed approaches of LDOs, focusing on the improving the stability and transient response, as well as the most recent work-simpler LDO structure, which is the contribution of this paper.

\section{ACKNOWLEDGMENT}

The authors would like to thank S. F. Luk for his technical support.

\section{REFERENCES}

[1] J. Ramirez-Angulo, A. Torralba, J. Galan, A. P. Vega-Leal, and J. Tombs, "Low-power low-voltage analog electronic circuits using the flipped voltage follower," in Proc. IEEE Int. Symp. Ind. Electron., Jul. 2002, pp. 1327-1330.

[2] J. Ramirez-Angulo, R. G. Garvajal, A. Torralba, J. Galan, A. P. VegaLeal, and J. Tombs, "The flipped voltage follwer: A useful cell for low-voltage low-power circuit design," in Proc. IEEE Int. Sym. Circuits Syst., May 2002, vol. 3, pp. 615-618.

[3] J. Ramirez-Angulo, S. Gupta, I. Padilla, R. G. Garvaja, A. Torralba, M. Jimenez, and F. Munoz, "Comparison of conventional and new flipped voltage structures with increased input/output signal swing and current sourcing/sinking capabilities," in Proc. IEEE Int. Sym. Circuits Syst., Aug. 2005, vol. 2, pp. 1151-1154.

[4] T. Y. Man, C. Y. Leung, K. N. Leung, P. K. T. Mok, and M. Chan, "Single-Transistor-Control Low-Dropout Regulator," U.S. Patent \# 7285952 , Oct. 23, 2007.

[5] G. A. Rincon-Mora and P. E. Allen, "Optimized frequency-shaping circuit topologies for LDO's," IEEE Trans. Circuits Syst. II, Analog Digit. Signal Process., vol. 45, no. 6, pp. 703-708, Jun. 1998.

[6] R. K. Dokania and G. A. Rincon-Mora, "Cancellation of load regulation in low drop-out regulators," Electron. Lett., vol. 38, no. 22, pp. 1300-1302, Oct. 2002.

[7] K. N. Leung and P. K. T. Mok, "A capacitor-free CMOS low-dropout regulator with damping-factor-control frequency compensation," IEEE J. Solid-State Circuits, vol. 38, no. 11, pp. 1691-1702, Oct. 2003.
[8] S. K. Lau, P. K. T. Mok, and K. N. Leung, "A low-dropout regulator for SoC with Q-reduction," IEEE J. Solid-State Circuits, vol. 42, no. 3 , pp. 658-664, Mar. 2007.

[9] C. K. Chava and J. Silva-Martinez, "A frequency compensation scheme for LDO voltage regulators," IEEE Trans. Circuits Syst. I, Reg. Papers, vol. 51, no. 6, pp. 1041-1050, Jun. 2004.

[10] W.-J. Hung, S.-H. Lu, and S.-I. Liu, "CMOS low dropout linear regulator with single Miller capacitor," Electron. Lett., vol. 42, no. 4, pp. 216-217, Feb. 2006.

[11] P. R. Gray, P. H. Hurst, S. H. Lewis, and R. G. Meyer, Analysis and Design of Analog Integrated Circuits, 4th ed. New York: Wiley, 2001.

[12] M. Pulkin and G. A. Rincon-Mora, "Stable Low Dropout, Low Impedance Driver for Linear Regulators," U.S. \#2003/0001550, Jan. 2, 2003.

[13] P. Hazucha, T. Kamik, B. A. Bloechel, C. Parsons, and S. Borkar, "Area-efficient linear regulator with ultra-fast load regulation," IEEE J. Solid-State Circuits, vol. 40, no. 4, pp. 933-940, Apr. 2005.

[14] E. Rogers, "Stability analysis of low-dropout regulators with a pMOS pass elements," Texas Instrum. Anal. Appl. J., pp. 10-12, Aug. 1999.

[15] B. S. Lee, "Technical Review of Low Dropout Regulator Operation and Performance," Texas Instruments, Dallas, Texas Instruments Appl. Rep. SLVA072, Aug. 1999.

[16] B. M. King, "Understanding the load-transient response of LDOs," Texas Instrum. Anal. Appl. J., pp. 19-23, Nov. 1999.

[17] S. K. Lau, K. N. Leung, and P. K. T. Mok, "Analysis of low-dropout regulator topologies for low-voltage regulation," in Proc. Conf. Electron Devices Solid-State Circuits, 2003, pp. 379-382.

[18] K. Salmi, C. Sarabello, O. Chevalerias, and F. Rodes, "4 V, 5 mA Low drop-out regulator using series-pass N-channel MOSFET," Electron. Lett., vol. 35, no. 15, pp. 1214-1215, Jul. 1999.

[19] D. Heisley and B. Wank, "DMOS delivers dramatic performance gains to LDO regulators," $E D N$, pp. 141-150, Jun. 2000.

[20] G. A. Rincon-Mora and P. E. Allen, "A low-voltage, low quiescent current, low drop-out regulator," IEEE J. Solid-State Circuits, vol. 33, no. 1, pp. 36-44, Jan. 1998.

[21] "Cap-Free, nMOS, 150 mA Low Dropout Regulator With Reverse Current Protection," Texas Instruments, Dallas, TPS731xx family, 2007.

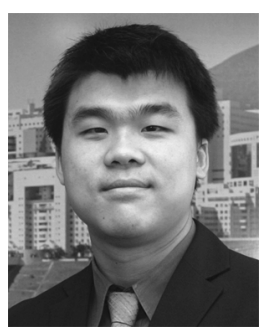

Tsz Yin Man (S'01) received the B.Eng. (highest honors), M.Phil., and Ph.D. degrees in electrical and electronic engineering from the Hong Kong University of Science and Technology, Hong Kong, in 2001, 2003, and 2008, respectively.

He is currently with Marvell Hong Kong Ltd, Hong Kong.

Mr. Man received the first prize in the 2001 IEEE Hong Kong student paper contest.

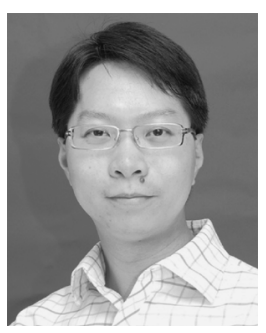

Ka Nang Leung (S'02-M'03-SM'08) received the B.Eng., M.Phil., and Ph.D. degrees, in electrical and electronic engineering, from The Hong Kong University of Science and Technology, Hong Kong.

$\mathrm{His} \mathrm{Ph} . \mathrm{D}$. research area is power-management integrated circuits in CMOS technology. He joined the Department of Electronic Engineering, The Chinese University of Hong Kong, in September 2005, as an Assistant Professor. He was a Visiting Assistant Professor in the Department of Electrical and Electronic Engineering, The Hong Kong University of Science and Technology. His current research interests include power-management integrated circuits for wireless telecommunication, biomedical filter design and CMOS image sensor. In addition, he is a technical paper reviewer of IEEE journals and international conferences.

In 1996, Prof. Leung received a Best Teaching Assistant Award from the Department of Electrical and Electronic Engineering, Hong Kong University of Science and Technology. In 2007, he received the Faculty and Department Exemplary Teaching Awards, The Chinese University of Hong Kong. He was the recipient of the 2003 Young Scientist Awards of the Hong Kong Institution of Science. 


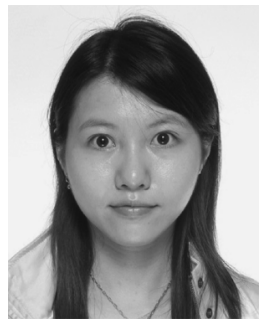

Chi Yat Leung received the B.Eng. and M.Phil. degrees in electrical and electronic engineering from the Hong Kong University of Science and Technology, Hong Kong.

She is currently a Research Assistant in Department of Electronic Engineering, The Chinese University of Hong Kong. She was a Memory Engineer in Fujitsu, Hong Kong. Her current interests include flash memory design, low-voltage current-sensing power converters, voltage references and low-dropout linear regulators.

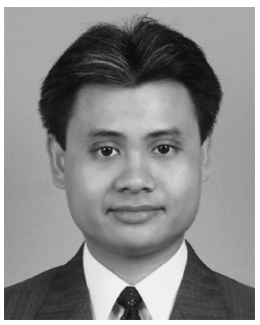

Philip K. T. Mok (S'86-M'95-SM'02) received the B.A.Sc., M.A.Sc., and Ph.D. degrees in electrical and computer engineering from the University of Toronto, Toronto, ON, Canada, in 1986, 1989, and 1995, respectively.

In January 1995, he joined the Department of Electronic and Computer Engineering, The Hong Kong University of Science and Technology, Hong Kong, China, where he is currently an Associate Professor. His research interests include semiconductor devices, processing technologies and circuit designs for power electronics and telecommunications applications, with current emphasis on power management integrated circuits, low-voltage analogue integrated circuits and RF integrated circuits design.

Dr. Mok received the Henry G. Acres Medal, the W.S. Wilson Medal and a Teaching Assistant Award from the University of Toronto, and the Teaching Excellence Appreciation Award twice from The Hong Kong University of Science and Technology. He is also a co-recipient of the Best Student Paper Award in the 2002 IEEE Custom Integrated Circuits Conference. In addition, he has been a member of the International Technical Program Committees of the IEEE International Solid-State Circuits Conference (ISSCC) since 2005 and he has served as an Associate Editor of IEEE TRANSACTIONS ON CIRCUITS AND SYSTEMS-II: EXPRESS BRIEFS from 2005 to 2007, the IEEE JOURNAL OF SOliD-STATE CirCUITS since 2006, and IEEE TRANSACTIONS ON CiRCUITS AND SYSTEMS-I: REGULAR PAPERS since 2007.

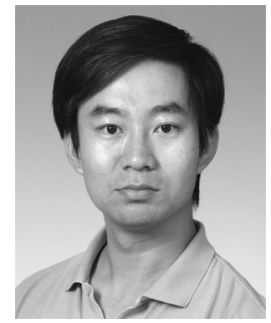

Mansun Chan (S'92-M'95-SM'01) received the B.S. degree in electrical engineering (highest honors) and B.S. degree in computer sciences (highest honors from University of California at San Diego, ) in 1990 and 1991 respectively, and the M.S. and Ph.D degrees degree from the University of California at Berkeley, in 1994 and 1995, respectively.

During his undergraduate study, he was working with Rockwell International Laboratory on Heterojunction Bipolar Transistor (HBT) modeling, where he developed the self-heating SPICE model for HBT His research at Berkeley covered a broad area in silicon devices ranging from process development to device design, characterization, and modeling. A major part of his work was on the development of record breaking silicon-on-insulator (SoI) technologies. He has also maintained a strong interest in device modeling and circuit simulation. He is one of the major contributors to the unified BSIM model for SPICE, which has been accepted by most U.S. companies and the Compact Model Council (CMC) as the first industrial standard MOSFET model. In January 1996, he joined the Electrical and Electronic Engineering faculty at Hong Kong University of Science and Technology, Hong Kong. His research interests include nanodevice technologies, image sensors, SOI technologies, high-performance integrated circuits, 3-D Circuit Technology, device modeling and Nano BioNEMS technology. Between July 2001 and December 2002, he was a Visiting Professor at University of California at Berkeley and the Co-director of the BSIM program. He is currently still consulting on the development of the next generation compact models.

Dr. Chan is a recipient of the University of California Regents Fellowship, Golden Keys Scholarship for Academic Excellence, SRC Inventor Recognition Award, Rockwell Research Fellowship, R\&D 100 award (for the BSIM3v3 project), Teaching Excellence Appreciation award (1999), Distinguished Teaching Award (2004) and other awards. 